\title{
Assessing and Treating Work-Related Asthma
}

\author{
Tracy Stoughton, MD, Michael Prematta, MD, and Timothy Craig, DO
}

\begin{abstract}
Work-related asthma is asthma that is caused or exacerbated by exposures at work. It is the most common form of occupational lung disease in developed countries. It has important impacts on the health and well-being of the affected individual, as well as consequences for society because of unemployment issues and workers' compensation claims. With ongoing exposure, occupational asthma can result in persistent airway hyperresponsiveness and, possibly, permanent disability for the individual. Thus, it is important for the clinician to be able to diagnose this disorder as quickly and accurately as possible. The evaluation of a patient with workrelated asthma can be extensive. It includes obtaining a consistent history, identifying the cause in the workplace, and confirming the diagnosis with objective tests. After a diagnosis has been made, treatment must sometimes go beyond the medications used for nonoccupational asthma and include interventions to minimize or completely remove the individual from exposure to the causal agent if he or she has sensitizer-induced occupational asthma. In addition, once an individual has been identified with occupational asthma, steps should be taken to prevent the development of this disorder in other workers. The purpose of this article is to review the current literature and provide the clinician with a stepwise approach to the diagnosis and management of a patient with work-related asthma.
\end{abstract}

Key words: airways, asthma, diagnosis, irritant, occupational, review, sensitizer, treatment, work

$W$ ork-related asthma is defined as asthma that is either attributable to or exacerbated by workplace environmental exposures. It encompasses two separate but related clinical entities: occupational asthma (OA) and workaggravated asthma (WAA). ${ }^{1}$ In both circumstances, the work environment contributes to the symptoms of asthma. However, $\mathrm{OA}$ is defined as asthma that is actually caused by the workplace, whereas WAA is classified as pre-existing asthma with symptoms aggravated by the work environment. ${ }^{1-3}$

OA has become increasingly important as it is now the most common occupational lung disorder in developed countries. Various studies have been performed to estimate the true prevalence of OA asthma in several countries. In Canada, estimated work-related asthma cases range from $42 /$ million/ year for women to $79 /$ million/year for men. ${ }^{4}$ In the United States, it has been estimated that anywhere between 3 and $29 \%$ of adult asthma can be attributed to occupational exposures. ${ }^{5-7}$ A large study performed in Europe in 1999 estimated that 5 to $10 \%$ of asthma in young adults could be ascribed to their occupations. ${ }^{8}$ Throughout most of these studies, certain

\footnotetext{
Tracy Stoughton: Department of Pediatrics, Michael Prematta: Department of Internal Medicine, and Timothy Craig: Department of Allergy and Immunology, Milton S. Hershey Medical Center, Hershey, PA.

Correspondence to: Timothy Craig, Allergy/Medicine, Penn State University, 500 University Dr., HO41, Hershey, PA 17033

(C) The Canadian Society of Allergy, Asthma and Clinical Immunology
}

DOI 10.2310/7480.2008.00020 occupations are consistently identified as having higher risk than others and include the wood industry, agriculture, food industry, painters, automobile industry, public administration (including police and firefighters), health services, and, in some studies, hairdressers. ${ }^{4,7-10}$

The effects of $\mathrm{OA}$ are numerous. In addition to the immediate health consequences, many studies have demonstrated the persistence of asthma symptoms, bronchial hyperresponsiveness, and airway inflammation even after removal from the causal agent (Figure 1). ${ }^{11-14}$ A study performed by Yacoub and colleagues found evidence that individuals with OA may also be at higher risk for psychological effects, in particular, anxiety disorders and dysthymia. ${ }^{14}$ Financial concerns for the individual are significant as well, with the majority of employees having to find alternative jobs after their diagnosis (many reporting a reduction of income), and as many as 25 to $30 \%$ of patients remaining unemployed after diagnosis. ${ }^{11,15}$ Finally, the effects of OA can be felt in the use of health resources (physician and emergency department visits), workers' compensation claims, and company changes that are undertaken to reduce the harmful exposures to their workers. $^{2,12}$

Because of the financial burden, health of the index case, risk to other workers with similar exposure, and prevalence of this condition, it becomes increasingly important for physicians to have a systematic way of evaluating patients for OA. Once the diagnosis is made, physicians must treat the patient and also give recommendations for interventions to be done at work. This may even include mobilizing consultants such 


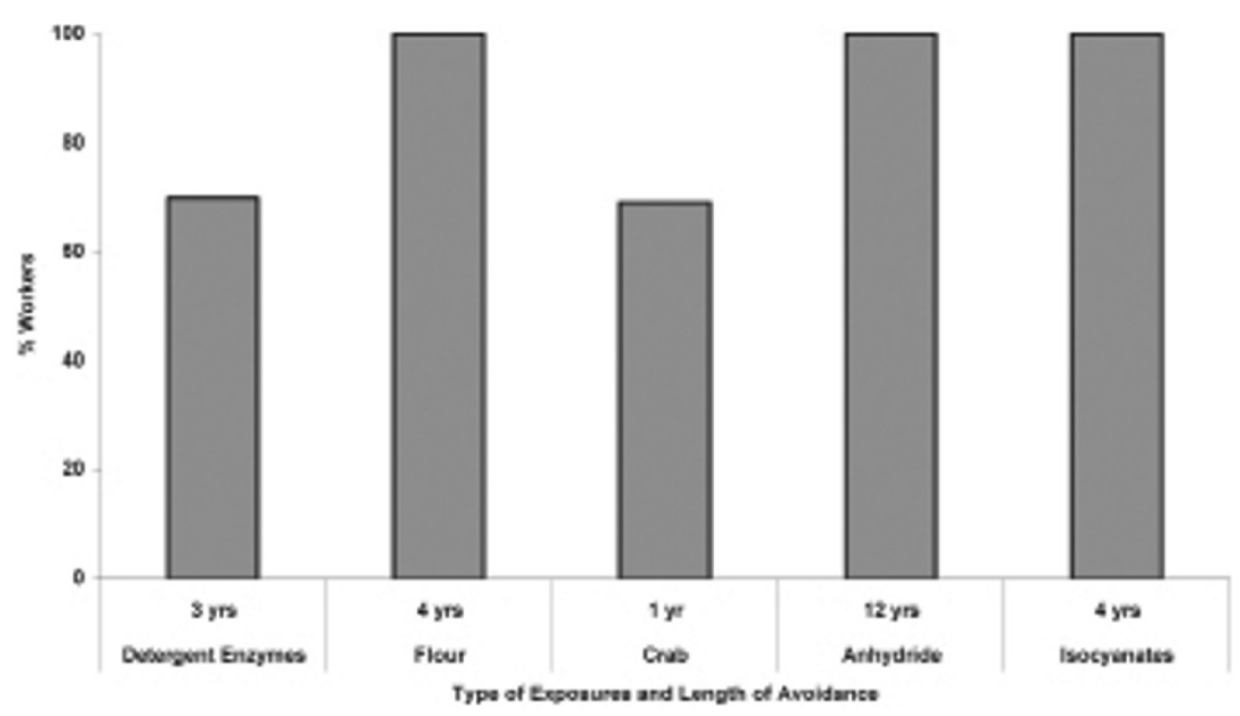

Figure 1. Percentage of workers with persistent symptoms following avoidance. Adapted from Brant A et al. ${ }^{11}$ as industrial hygienists to assess the workplace and advise on proper adaptations that can protect the workforce.

\section{Methods}

OVID and PubMed were searched using the terms occupational asthma, work-aggravated asthma, irritant-induced asthma, and reactive airway dysfunction syndrome (RADS) alone and in combination with epidemiology, diagnosis, and treatment. Both primary literature and recent reviews were included to create a document that can be used by the practicing allergist to assess and treat patients who may have workrelated asthma.

\section{Classification of Work-Related Asthma}

Work-related asthma can be triggered by different types of compounds and mediated by a variety of mechanisms. Specifically, OA can be immunologically mediated by both high(HMW) and low-molecular-weight (LMW) substances and may also be non-immunologically mediated irritant-induced asthma or RADS. WAA occurs in those with pre-existing asthma in response to a variety of different triggers found in the workplace, whereas $\mathrm{OA}$ is directly induced by workplace exposure.

HMW agents, animal and plant proteins, cause OA through the induction of specific IgE antibodies and the typical cascade of events that are seen in "allergic asthma"; however, specific IgE may be present even in those without asthma. The pathophysiology of this type of OA is similar to non-occupational allergic asthma. ${ }^{1,3}$ HMW substances can result in direct sensitization and thereby cause OA or can ex- acerbate pre-existing asthma. Rarely, they may also induce isolated late responses or atypical reactions.

LMW chemicals also can lead to OA by immunologic mechanisms, resulting in airway inflammation with eosinophils, lymphocytes, mast cells, and thickened reticular basement membranes. ${ }^{3}$ However, although some LMW agents, such as platinum salts and trimellitic anhydride, cause OA via specific IgE antibodies similar to HMW antigens, other LMW agents, such as plicatic acid in red cedar and diisocyanates, do not consistently result in the production of specific IgE antibodies. These agents may cause a variety of results with specific inhalation challenges, including immediate and dual reactions, as well as late asthmatic or atypical reactions. ${ }^{3}$ There are currently several hypotheses about the pathophysiology of these apparently IgE-independent reactions, but further research is required to delineate the true mechanism. ${ }^{3,16}$ Regardless of the exact pathophysiology, all of these types of $\mathrm{OA}$ are similar in that the patients who are sensitized to these agents will have further exacerbation of their symptoms and airway inflammation if exposure continues and are likely to have persistence of airway hyperresponsiveness even after removal from the offending agent following prolonged exposure. $^{2}$

OA can also be mediated by a yet unknown immunologic process, and since previous exposure is not necessary, it is often thought to be an irritant-induced mechanism. RADS is asthma in a previously healthy individual caused by a single high-level exposure to an airway irritant. According to the American College of Chest Physicians, the diagnosis of RADS should include the following: "(1) a documented absence of preceding respiratory complaints; (2) onset of symptoms after a single exposure incident or accident; (3) exposure to a 
gas, smoke, fume, or vapor with irritant properties present in very high concentrations; (4) onset of symptoms within $24 \mathrm{~h}$ after the exposure with persistence of symptoms for at least 3 months; (5) symptoms simulate asthma with cough, wheeze, and dyspnea; (6) presence of airflow obstruction on pulmonary function tests; (7) presence of nonspecific bronchial hyperresponsiveness; and (8) other pulmonary diseases ruled out." ${ }^{17}$ RADS can be caused by many different agents, such as chlorine, diisocyanates, smoke inhalation, phosphoric acid, sulphuric acid, and ammonia. ${ }^{18,19}$ Of note, some of these agents can also be sensitizers. The proposed pathophysiology of RADS involves direct epithelial damage, with resultant neurogenic inflammation. ${ }^{3,18}$

Some researchers have proposed expanding the definition of RADS to include more than one short-term high-level exposure and onset of symptoms up to 7 days after exposure; others go even further to include chronic exposure to low levels of irritants. However, these suggestions have not been widely accepted as an expansion of the definition and would make it even more difficult to differentiate RADS from other causes of airway hyperresponsiveness. ${ }^{18,19}$

WAA is the final category of work-related asthma. In WAA, patients with known asthma can have exacerbations caused by inhalation of irritants at work (aerosols, dust, gases, fumes) or worsening symptoms caused by cold air exposure or exertion. ${ }^{2,20}$

\section{Identify Exposures}

When first interviewing a patient with suspected work-related asthma, it is important to identify exposures in the workplace that might be causing or contributing to their symptoms. It is necessary to understand their current job activities, with particular emphasis on processes that transfer materials, disturb allergen reservoirs, and form new reaction products. ${ }^{17}$ Clinicians should also get a sense of nearby processes and the "intensity of airborne exposures by asking about visible dust, odors, and mucous membrane irritation." ${ }^{2}$ Specific inquiries should also be made about known triggers of asthma, including cold air, exertion, pollens, animal dander, mould, fumes, cigarette smoke, vapours, and ambient air pollution., ${ }^{2,3}$

In addition to the patient interview, exposure risk can be assessed from other sources of information at the workplace itself. Material safety data sheets (MSDSs) can be a very useful source of information. The MSDSs list the name, manufacturer, and chemical composition of toxic agents in the workplace and describe the potential health effects of these agents. In this way, they can be very useful for helping to identify potential sensitizing agents. ${ }^{2,17}$ However, it should be noted that
MSDSs can sometimes be incomplete as they do not always give information on sensitizers if they are found in low concentrations, even if they can be harmful. ${ }^{21}$ With the patient's consent, information can also be obtained from people in the workplace, such as the employer, management, and medical personnel. Some companies may also employ industrial hygiene specialists who are responsible for recognizing, measuring, and controlling workplace exposures. If available, these personnel can assist in identifying agents that may be causing or playing a role in a patient's work-related asthma. ${ }^{2,17}$ Finally, if feasible, a walk-through performed by the clinician may also be valuable in identifying possible sensitizers. ${ }^{17}$

\section{Gather Clinical Evidence}

When evaluating a patient for work-related asthma, obtaining a clinical history is the first step in gathering supportive evidence. In addition to obtaining exposure risks as noted above, it is important to obtain a complete past medical history with emphasis on childhood respiratory symptoms, history of atopy, previous home and work exposures, and cigarette smoking. ${ }^{17,22}$ It is crucial to understand the patient's current symptoms, as well as their progression, since many times OA begins with upper airway symptoms such as rhinorrhea, congestion, and sneezing and progresses to chest tightness, cough, wheezing, and dyspnea. ${ }^{2,17,22}$ In addition, any temporal relationship to their current profession, that is, initial onset of symptoms (which can vary from months to years from initial employment), worsening symptoms while at work, and improved symptoms while away from work, should be ascertained (Figure 2). ${ }^{2,3,17}$ Unfortunately, even a history that appears to be consistent with $\mathrm{OA}$ is not sufficient to diagnose OA. Malo and colleagues demonstrated that the clinical history has a positive predictive value of only $63 \%$ but a negative predictive value of $83 \%$ when compared with specific inhalation challenges. ${ }^{23}$ Thus, the clinical history is more useful in helping rule out $\mathrm{OA}$ than in ruling in $\mathrm{OA}$.

In the initial diagnostic workup of $\mathrm{OA}$, immunologic testing in the form of skin-prick testing, radioallergosorbent test (RAST), or enzyme-linked immunosorbent assay can be considered. ${ }^{2}$ As mentioned above, many different mechanisms lead to the development of OA, and, unfortunately, not all are consistently driven by specific IgE antibodies, which makes it difficult to reliably diagnose all forms of $\mathrm{OA}$ via immunologic tests. In addition, these tests are often limited by commercial availability and a lack of standardized agents. ${ }^{3,24}$ Despite these limitations, skin-prick tests can be useful in the diagnosis of OA caused by HMW agents (eg, animal proteins, wheat). Thus, when available, skin-prick testing 


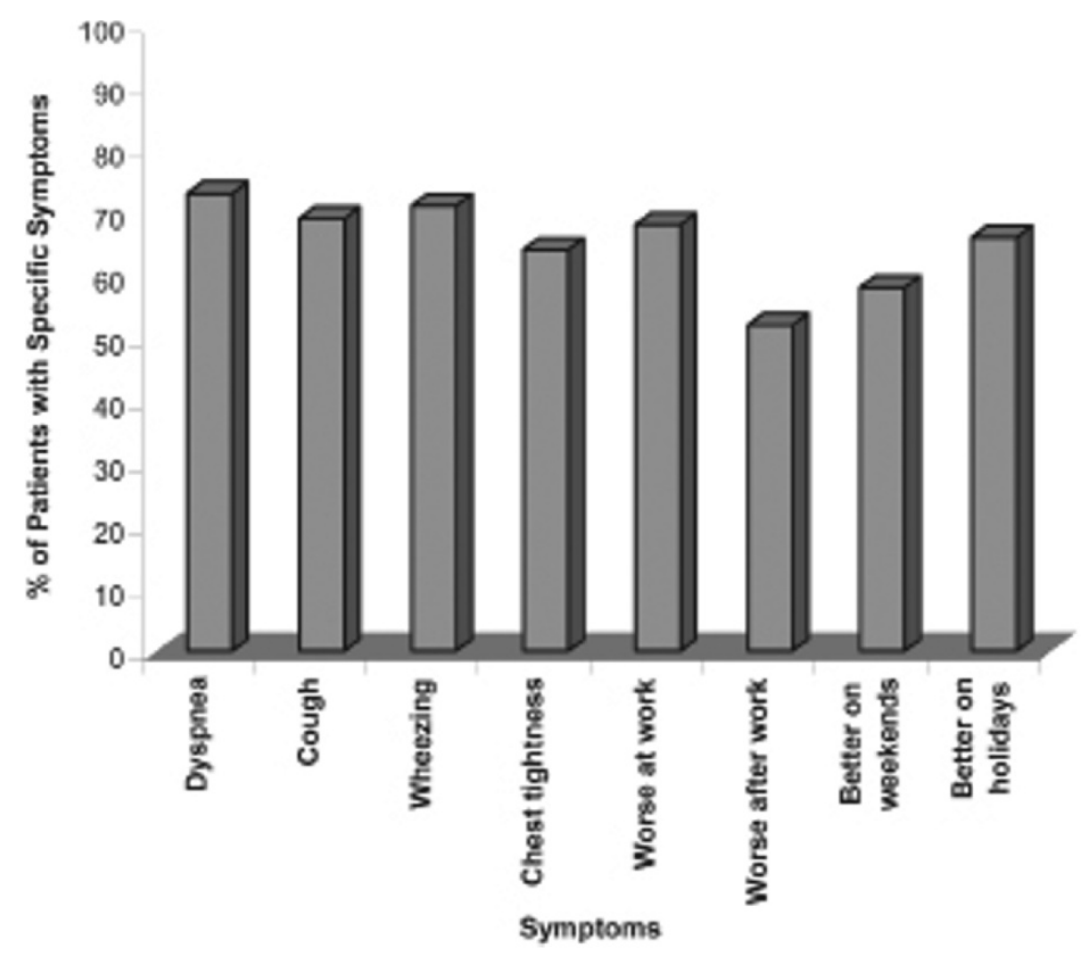

Figure 2. Symptomatic presentation of occupational asthma. Adapted from Malo J-L et al. ${ }^{23}$

is generally recommended in the workup of OA caused by these agents. ${ }^{17,25}$ With few exceptions, immunologic testing for LMW agents is more difficult since many of these agents do not consistently result in the production of specific IgE, and positive serum IgE can also be indicative of exposure but not necessarily disease. ${ }^{3}$ However, a study done on isocyanates did show that a positive serum IgE, indicated by a RAST score of 3 or greater, was highly specific (100\%) for the diagnosis of isocyanate-induced OA. Therefore, its presence, along with a consistent clinical history, was diagnostic for isocyanate-induced OA. Unfortunately, the sensitivity of this test was only $20 \%$, so it cannot be reliably used to diagnose all cases of isocyanate-induced OA. ${ }^{26}$

A second test that can be used in the workup of $\mathrm{OA}$ is the measurement of nonspecific bronchial hyperreactivity (NSBH), most commonly assessed through methacholine challenge $(\mathrm{MC})$ tests, performed either serially or as a single initial test. In a recent systematic review, use of a single NSBH test was found to have a pooled sensitivity of $79.3 \%$ and a specificity of $51.3 \%$ when compared with specific chamber challenge. ${ }^{27}$ Similar findings in other studies ${ }^{28}$ have led some to recommend checking $\mathrm{MC}$ as part of the initial workup of OA, essentially to help establish the diagnosis of asthma. ${ }^{24,27}$ Then, after bronchial hyperreactivity has been confirmed, the physician can proceed with testing that actually links asthma to the exposures at work. On the other hand, some research- ers have studied serial MC for the primary diagnosis of OA. To do this, MC is performed at the end of an extended work period, preferably within 24 hours of work, and then again at the end of an extended period (some suggest 3 weeks) of leave from work. A threefold or greater increase in the provocative concentration dose required to reduce forced expiratory volume in 1 second $\left(\mathrm{FEV}_{1}\right)$ by $20 \%$, while away from work, is considered to be consistent with OA. ${ }^{20,24}$ Unfortunately, not all researchers have found benefit in serial MC when compared with serial peak expiratory flow (PEF) rates and specific challenge tests, ${ }^{29,30}$ so use of this test should generally be in conjunction with other clinical evidence.

Another method commonly used to evaluate OA is monitoring serial PEF rates in and out of the workplace. Patients should ideally monitor PEF every 2 hours while awake (three readings each time) for a period of 4 weeks - preferably 2 weeks at work, if tolerated, and 2 or more weeks away from work. ${ }^{29,31}$ During this time period, patients should also keep a diary of their activities with specific details on working times and activities. ${ }^{31}$ As this regimen can create difficulties with compliance, some have recommended measurement of PEF less frequently during the day. If this is considered, measurements should still be taken at least four times per day to preserve sensitivity and specificity. ${ }^{32}$ The values are then submitted for analysis. Several different methods of analysis exist. Most commonly, values are plotted and visually examined 
for variations between PEF measurements at and away from work. Visual examination of serial PEF by experts produces a sensitivity and a specificity between 73 and $86 \%$ and 74 and $100 \%$, respectively, when compared with specific chamber challenge. ${ }^{29,30,33}$ In addition, a computer program, OASYS-2 (Oasys Research Group, Midland Thoracic Society, UK), has been designed to analyze PEF variations without the use of graphs or expert interpretation. This computer program has been found to have a sensitivity of $69 \%$ and a specificity of $94 \%$ in people with a diagnosis of OA made independently of serial PEF. ${ }^{34}$ Of note, although some have hypothesized that serial measurement of $\mathrm{FEV}_{1}$ would be superior to serial PEF, a study performed by Leroyer and colleagues did not find this to be true; in fact, this study showed that analysis of serial PEF measurements was more sensitive and specific than analysis of serial $\mathrm{FEV}_{1}{ }^{33}$ This study also demonstrated superior results using the best of three values for analysis as opposed to the common method of using the best of two reproducible values.

PEF monitoring is popular owing to the fact that it has relatively high sensitivity and specificity, is easy to perform, and is inexpensive. It also gives measurements over time, increasing the likelihood of including late reactions and prolonged recoveries. Unfortunately, despite these benefits, this method also has several drawbacks: it is a time-consuming test and thus creates difficulties with patient compliance; it is effort dependent, and since it is an unsupervised test, the patient must be relied on to use best effort with each measurement; it can underestimate changes in airway calibre; it does not identify the causal agent but just a relationship between work and changes in PEF; and finally, concern arises regarding falsification of records, especially when compensation is at stake. ${ }^{17,31}$ Fortunately, if falsification is a serious concern, computerized peak flow meters are available and can be used to eliminate the concern of writing down false numbers. ${ }^{17,31}$ However, these meters still cannot evaluate patient effort.

Another test that can be used in the objective evaluation of $\mathrm{OA}$ is analyzing the number of eosinophils in induced sputum. In this form of testing, sputum production is induced using increasing concentrations of inhaled hypertonic saline during periods at and away from work. ${ }^{35,36}$ It has been found that patients with OA have higher numbers of sputum eosinophils and eosinophil cation protein during periods at work compared with periods away from work. ${ }^{36}$ In a study performed by Girard and colleagues, an increase in sputum eosinophils of $2 \%$ was found to add to the sensitivity and specificity of PEF monitoring by $8.2 \%$ and $18 \%$, respectively. ${ }^{35}$ Thus, it appears that the use of induced sputum may be a valuable adjunct to other tests in the assessment of OA.

Finally, OA can be assessed with the use of specific inhalation challenge testing, which is considered the gold standard for the diagnosis of OA. The exact procedure for a specific challenge depends on the type of agent being investigated. Some agents can be safely aerosolized and delivered via nebulizer for specific challenge testing. For these agents, concentration of the agent can be increased on different testing days until a decrease in $\mathrm{FEV}_{1}$ by $20 \%$ is achieved, which is considered a positive provocative challenge. ${ }^{25}$ In the assessment of OA caused by vapours, fumes, or gases, testing may require a challenge chamber. A challenge chamber is a room or a small, enclosed space where the suspected agent is delivered into the atmosphere at precise concentrations. ${ }^{25,37}$ Care is taken not to exceed the concentration of the agent that would be encountered at work or a concentration that could be considered an irritant. ${ }^{25,37}$ Finally, for some agents, innovative ways must be designed to safely test for sensitization.

The advantage of specific inhalation challenge testing is that it can provide a definitive answer, both confirming the diagnosis of OA and identifying the causal agent. Unfortunately, there are multiple drawbacks to specific inhalation testing. There is a lack of standardization for this type of testing. Therefore, it must be performed in specialized laboratories with the appropriate equipment and a general familiarity with effective, safe testing doses. ${ }^{37}$ False-positive results can occur in patients who have unstable asthma or are exposed to irritant levels of the agent. ${ }^{20}$ For patients who are exposed to multiple potential sensitizers, testing may have to be done to a number of different agents to achieve a diagnosis. ${ }^{37}$ Even if multiple specific challenges are performed, false-negative results can still occur if testing with the correct agent is not performed ${ }^{27}$ or if the exposure during testing is not of adequate concentration or duration. ${ }^{20}$ Given that late reactions can also occur, pulmonary function monitoring must continue for at least 6 hours, if not longer, after the exposure has taken place to avoid missing the diagnosis in those with isolated late reactions. ${ }^{20,37}$ Lastly, some have advocated the use ofMC tests on the day before and after specific inhalation testing to increase the sensitivity of this test. Unfortunately, there are only a few large academic centres in Canada and the United States where specific challenges can be performed successfully and safely.

In summary, none of the available objective tests are perfect for diagnosing OA. As a result, cases should be considered on an individual basis and tests should be ordered accordingly, with consideration of the benefits and drawbacks of each. Often a combination of tests performed in a stepwise approach, along with a consistent history, is most helpful in the diagnosis of OA. Even though specific inhalation challenges are considered the gold standard, they are difficult to perform and do not necessarily provide a definitive diagnosis. For these reasons, the current general recommendation 
Table 1. Essential Literature for the Practicing Allergist

\begin{tabular}{lll}
\hline Article Name & Author(s) & Journal \\
\hline $\begin{array}{l}\text { "Canadian Thoracic Society Guidelines for } \\
\text { Occupational Asthma" }\end{array}$ & $\begin{array}{c}\text { Susan M. Tarlo, Louis-Philippe Boulet, } \\
\text { André Cartier, et al }\end{array}$ & Can Respir J 1998;5:289-300 \\
"Assessment of Asthma in the Workplace. ACCP & M. Chan-Yeung & Chest 1995;108:1084-117 \\
Consensus Statement" & & \\
"Guidelines for Assessing and Managing Asthma & American Thoracic Society & Am J Respir Crit Care Med 2004; \\
Risk at Work, School, and Recreation" & 169:873-81 \\
"Evidence Based Guidelines for the Prevention, & P.J. Nicholson, P. Cullinan, A.J. Newman \\
Identification, and Management of & Occup Environ Med 2005;62:290-9 \\
Occupational Asthma" & & \\
\hline
\end{tabular}

is that specific inhalation challenges are not essential for the diagnosis of OA. However, they should be considered in cases in which the diagnosis of OA cannot be accomplished with other tests. ${ }^{20}$

\section{Interventions}

Pharmacologic treatment for work-related asthma does not differ from that for non-OA. ${ }^{17}$ Inhaled corticosteroids are used for chronic therapy and acute symptoms treated with bronchodilators. In addition, patients should be educated about their asthma, taught to recognize and treat symptoms appropriately, and have asthma action plans. Patients should also avoid non-occupational exposures that exacerbate their asthma symptoms, such as smoking and allergen exposure if applicable. ${ }^{20}$

Unfortunately, since work-related asthma is caused or worsened by exposures encountered at work, interventions have to go beyond those normally taken for non-OA. Patients must be counselled about their ongoing risks if exposure to the offending agent continues. For OA induced by sensitizing agents, the prognosis is worsened by longer periods of exposure to the agent. Even minute exposures can induce symptoms, and patients can retain their sensitivity to these agents even if they have been unexposed and asymptomatic for prolonged periods. ${ }^{13}$ Thus, the goal for patients with OA induced by sensitizing agents is to be removed from all exposures to the offending agent. ${ }^{17,20}$ They should also avoid reexposure in the future. ${ }^{13}$ For patients who have irritant-induced or worsened asthma, either RADS or WAA, it may not be necessary to completely avoid all exposure to the offending agent but rather to ensure that high levels of the agent are not encountered. ${ }^{17,20}$

Several different steps can be taken at the workplace to accommodate for patients with work-related asthma. Companies can replace the identified sensitizing agent with another less sensitizing or toxic agent. Processes can be enclosed, or effective local ventilation systems can be established. If these are not practical options, the patient with sensitizer-induced OA can be transferred to a different job or location to ensure that they are no longer exposed to the agent. ${ }^{2,17,20}$ Finally, a respirator for personal protection can also be provided to patients with work-related asthma, particularly for short-term exposures. When used, it must be ensured that the respirator is the correct type and is fitted properly and that the patient is educated on how to wear and remove it. ${ }^{38}$ The longterm use of respirators in a patient with sensitizer-induced $\mathrm{OA}$ is generally not recommended as the patient may still continue to be exposed to small amounts of the agent, and even small amounts can trigger symptoms. For patients with irritant-induced asthma, the use of a respirator, on a periodic basis, may be adequate for control of their symptoms. ${ }^{2,17,20}$

After appropriate interventions have been taken to treat work-related asthma, it is important to continue monitoring the patient and exposed coworkers. Continued monitoring can help the clinician and the patient determine whether the accommodations are effective. ${ }^{2}$ This is particularly important in sensitizer-induced $\mathrm{OA}$, for which fatalities have been reported in patients who remain exposed. As mentioned previously, even patients who have been removed from exposure to the sensitizing agent or irritant can have persistence of bronchial hyperresponsiveness. Thus, it is important to continue monitoring these patients to ensure adequate symptom control. Finally, it is also important for the clinician to consider the psychological and financial impacts that OA may have on the patient. As anxiety and dysthymia have both been linked to OA, screening for these conditions can be an important adjunct to the patient's overall care. ${ }^{14}$ Clinicians caring for patients with OA should also be prepared to participate in workers' compensation claims and disability if necessary to provide the objective clinical evidence that was used for the diagnosis of $\mathrm{OA}$ and advocate for their patient to receive appropriate compensation if applicable. ${ }^{3}$

\section{Prevention and Surveillance}

Outcomes in sensitizer-induced OA are improved when patients have a shorter duration of symptoms prior to diagnosis, nearly normal lung function at the time of diagnosis, and 
early removal from the causative agent. Early identification and intervention are therefore crucial to improved medical and financial outcomes for the patient. As a result, recommendations have been made to help employers reduce the number of workers who develop OA and identify those who are affected as early as possible. In professions in which the risk of $\mathrm{OA}$ is high, these recommendations include removing or reducing exposures to known sensitizing agents for all workers and instituting surveillance programs in which employees at risk for work-related asthma are screened by health and safety personnel with questionnaires, pulmonary function tests, and immunologic tests, as appropriate. ${ }^{3,20,38}$ More frequent monitoring should be performed for workers who have underlying asthma or develop rhinitis. ${ }^{38}$ In addition, patients who develop positive immunologic reactions should also undergo more frequent monitoring and consider reducing exposures to the indicated agent at the time the sensitivity is discovered. ${ }^{20}$

\section{Summary}

In conclusion, work-related asthma is a common disorder that is increasing in prevalence and has important medical, social, and economic consequences. The causes of work-related asthma are vast and mediated by a variety of immunologic and non-immunologic mechanisms. As a result, the diagnosis of work-related asthma can be a complicated and timeconsuming task. In general, physicians should take a stepwise approach, starting with a comprehensive medical history and evaluation of exposures, and then proceed with objective tests to support the diagnosis. No one test is the definitive diagnostic test for work-related asthma, so a combination of tests is usually indicated. If the diagnosis of work-related asthma is established, steps must be taken to ensure adequate removal or minimization of the causal agent from the patient's work environment. As OA may be a progressive disease with worsening prognosis as exposure continues, early diagnosis and intervention are imperative for the patient. Importantly, each worker who develops OA should be considered an index case, and other exposed workers should be assessed to reduce the possibility that they develop OA. Optimally, prevention strategies and surveillance programs in the workplace should prevent the development and progression of OA; however, at the present time, these programs are inadequate to completely prevent occupation-induced diseases. The practicing allergist is encouraged to have the resources listed in Table 1 available to use as a reference in assessing a patient with work-related asthma.

\section{References}

1. Friedman-Jiménez G, Beckett WS, Szeinuk J, Petsonk EL. Clinical evaluation, management, and prevention of work-related asthma. Am J Ind Med 2000;37:121-41.

2. American Thoracic Society. Guidelines for assessing and managing asthma risk at work, school, and recreation. Am J Respir Crit Care Med 2004;169:873-81.

3. Mapp CE, Boschetto P, Maestrelli P, Fabbri LM. Occupational asthma. Am J Respir Crit Care Med 2005;172:280-305.

4. Provencher S, Labrèche FP, De Guire L. Physician based surveillance system for occupational respiratory diseases: the experience of PROPULSE, Québec, Canada. Occup Environ Med 1997;54:272-6.

5. Blanc P. Occupational asthma in a national disability survey. Chest 1987;92:613-7.

6. Sama SR, Milton DK, Hunt PR, et al. Case-by-case assessment of adultonset asthma attributable to occupational exposures among members of a health maintenance organization. J Occup Environ Med 2006;48: $400-7$.

7. Timmer S, Rosenman K. Occurrence of occupational asthma. Chest 1993;104:816-20.

8. Kogevinas M, Antó JM, Sunyer J, et al. Occupational asthma in Europe and other industrialised areas: a population-based study. Lancet 1999;353:1750-4.

9. Ameille J, Pauli G, Calastreng-Crinquand A, et al. Reported incidence of occupational asthma in France, 1996-99: the ONAP programme. Occup Environ Med 2003;60:136-41.

10. McDonald JC, Chen Y, Zekveld C, Cherry NM. Incidence by occupation and industry of acute work related respiratory diseases in the UK, 1992-2001. Occup Environ Med 2005;62:836-42.

11. Brant A, Zekveld C, Welch J, et al. The prognosis of occupational asthma due to detergent enzymes: clinical, immunological and employment outcomes. Clin Exp Allergy 2006;36:483-8.

12. Goe SK, Henneberger PK, Reilly MJ, et al. A descriptive study of work aggravated asthma. Occup Environ Med 2004;61:512-7.

13. Lemière C. Persistence of bronchial reactivity to occupational agents after removal from exposure and identification of associated factors. Ann Allergy Asthma Immunol 2003;90 Suppl:52-5.

14. Yacoub MR, Lavoie K, Lacoste G, et al. Assessment of impairment/ disability due to occupational asthma through a multidimensional approach. Eur Respir J 2007;29:889-96.

15. Ameille J, Pairon JC, Bayeux MC, et al. Consequences of occupational asthma on employment and financial status: a follow-up study. Eur Respir J 1997; 10:55-8.

16. Liu Q, Wisnewski AV. Recent developments in diisocyanate asthma. Ann Allergy Asthma Immunol 2003;90 Suppl:35-41.

17. Chan-Yeung M. Assessment of asthma in the workplace. ACCP consensus statement. Chest 1995;108:1084-117.

18. Bardana EJ. Reactive airways dysfunction syndrome (RADS): guidelines for diagnosis and treatment and insight into likely prognosis. Ann Allergy Asthma Immunol 1999;83:583-6.

19. Tarlo SM. Workplace irritant exposures: do they produce true occupational asthma. Ann Allergy Asthma Immunol 2003;90 Suppl:19-23.

20. Tarlo SM, Boulet L-P, Cartier A, et al. Canadian Thoracic Society guidelines for occupational asthma. Can Respir J 1998;5:289-300.

21. Bernstein JA. Material safety data sheets: are they reliable in identifying human hazards? J Allergy Clin Immunol 2002;110:35-8. 
22. Mihalas L. An approach to the diagnosis of occupational asthma. Ann Allergy Asthma Immunol 1999;83:577-82.

23. Malo J-L, Ghezzo H, L'Archevêque J, et al. Is the clinical history a satisfactory means of diagnosing occupational asthma? Am Rev Respir Dis 1991;143:528-32.

24. Tarlo SM, Liss GM. Occupational asthma: an approach to diagnosis and management. Can Med Assoc J 2003;168:867-71.

25. Tan RA, Spector SL. Diagnostic testing in occupational asthma. Ann Allergy Asthma Immunol 1999;83:587-92.

26. Tee RD, Cullinan P, Welch J, et al. Specific IgE to isocyanates: a useful diagnostic role in occupational asthma. J Allergy Clin Immunol 1998;101:709-15.

27. Beach J, Russell K, Blitz S, et al. A systematic review of the diagnosis of occupational asthma. Chest 2007;131:569-78.

28. Baur X, Huber H, Degens PO, et al. Relation between occupational asthma case history, bronchial methacholine challenge, and specific challenge test in patients with suspected occupational asthma. Am J Ind Med 1998;33:114-22.

29. Côté J, Kennedy S, Chang-Yeung M. Sensitivity and specificity of $\mathrm{PC}_{20}$ and peak expiratory flow rate in cedar asthma. J Allergy Clin Immunol 1990;85:592-8.

30. Perrin B, Lagier F, L'Archevêque J, et al. Occupational asthma: validity of monitoring of peak expiratory flow rates and non-allergic bronchial responsiveness as compared to specific inhalation challenge. Eur Respir J 1992;5:40-8

31. Anees W. Use of pulmonary function tests in the diagnosis of occupational asthma. Ann Allergy Asthma Immunol 2003;90 Suppl:47-51.

32. Malo JL, Côté J, Cartier A, et al. How many times per day should peak expiratory flow rates be assessed when investigating occupational asthma. Thorax 1993;48:1211-7.

33. Leroyer C, Perfetti L, Trudeau C, et al. Comparison of serial monitoring of peak expiratory flow and $\mathrm{FEV}_{1}$ in the diagnosis of occupational asthma. Am J Respir Crit Care Med 1998;158:827-32.

34. Bright P, Burge SP. Occupational lung disease 8: the diagnosis of occupational asthma from serial measurements of lung function at and away from work. Thorax 1996;51:857-63.

35. Girard F, Chaboillez S, Cartier A, et al. An effective strategy for diagnosing occupational asthma: use of induced sputum. Am J Respir Crit Care Med 2004;170:845-50.

36. Lemière C, Pizzichini MM, Balkissoon R, et al. Diagnosing occupational asthma: use of induced sputum. Eur Respir J 1999;13:482-8.

37. Pepys J, Hutchcroft BJ. Bronchial provocation tests in etiologic diagnosis and analysis of asthma. Am Rev Respir Dis 1975;112:829-59.

38. Nicholson PJ, Cullinan P, Newman Taylor AJ, et al. Evidence based guidelines for the prevention, identification, and management of occupational asthma. Occup Environ Med 2005;62:290-9. 